\title{
Vitamin D Deficiency and Sarcopenia in Hip Fracture Patients
}

\author{
Hong Seok Kim ${ }^{1, *}$, Geun Jang ${ }^{1, *}$, Jung Wee Park², Young-Kyun Lee' ${ }^{1}$ Kyung-Hoi Koo' \\ 'Department of Orthopaedic Surgery, Seoul National University Bundang Hospital, Seoul National University College of Medicine, Seongnam; \\ ${ }^{2}$ Department of Orthopaedic Surgery, Chung-Ang University College of Medicine, Seoul, Korea
}

Corresponding author

Young-Kyun Lee

Department of Orthopaedic Surgery, Seoul

National University Bundang Hospital, Seoul

National University College of Medicine,

82 Gumi-ro 173 beon-gil, Bundang-gu,

Seongnam 13620, Korea

Tel: +82-31-787-7204

Fax: +82-31-787-4056

E-mail: ykleemd@gmail.com

Received: November 20, 2020

Revised: December 13, 2020

Accepted: December 21, 2020

*Hong Seok Kim and Geun Jang contributed

equally to this work and should be

considered co-first authors.
Copyright $@ 2021$ The Korean Society for Bone and Mineral Research

This is an Open Access article distributed under the terms of the Creative Commons Attribution Non-Commercial License (https://creativecommons.org/licenses/by-nc/4.0/) which permits unrestricted non-commercial use, distribution, and reproduction in any medium, provided the original work is properly cited.

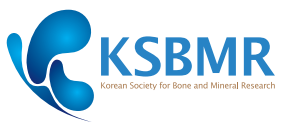

Background: Vitamin D plays a critical role in the proliferation and differentiation of skeletal muscle and bone metabolism. We compared the prevalence of vitamin $D$ deficiency in elderly patients undergoing hip fracture surgery (HFS) with those undergoing elective primary total hip arthroplasty (THA). We also evaluated the association between vitamin $D$ deficiency and sarcopenia. Methods: This prospective study included 70 patients in the HFS group, and 100 patients in the elective THA group. The HFS group included 42 women, while the elective THA group included 74 women. The mean age in the HFS and elective THA groups was 79.8 and 75.4 years, respectively, and the mean preoperative Koval score was 2.1 and 2.3, respectively. Serum 25-hydroxy-vitamin D3 levels were measured by 1125 radioimmunoassay. Bone mineral density and appendicular skeletal muscle mass (ASM) were measured using dual X-ray absorptiometry. Sarcopenia index was calculated by dividing ASM $(\mathrm{kg})$ by the body mass index $\left(\mathrm{kg} / \mathrm{m}^{2}\right)$. Sarcopenia was diagnosed when the sarcopenia index was $<0.789$ in men and $<0.512$ in women. Results: Serum vitamin D levels were lower, and the percentages of patients with vitamin $D$ insufficiency and deficiency were higher in the HFS group than in the elective THA group. In the HFS group, the prevalence of sarcopenia was higher in men $(57 \%, 16 / 28)$ than in women $(29 \%, 12 / 42 ; P=0.025)$. Conclusions: Vitamin D deficiency was more prevalent in patients undergoing HFS than in patients undergoing elective THA. Among patients undergoing HFS, vitamin D deficiency was more prevalent in sarcopenic than in nonsarcopenic patients.

Key Words: Hip fractures · Osteoporosis · Sarcopenia · Vitamin D

\section{INTRODUCTION}

Vitamin $\mathrm{D}$ is responsible for intestinal absorption of calcium, magnesium, and phosphate and has a critical role in bone metabolism. It also stimulates the proliferation and differentiation of skeletal muscles.[1]

Elderly people have a higher prevalence of low vitamin $D$ levels due to low dietary intake and reduced exposure to sunlight. Thus, elderly people with vitamin D deficiency are at risk of osteoporosis and sarcopenia.[2]

Osteoporotic fractures are worldwide health problems and socioeconomic burdens.[3] Hip fractures, which are the second most common fractures next to spine fractures, are critical causes of high morbidity and mortality in osteoporotic cohort.[3]

A recent multi-national epidemiologic study of postmenopausal osteoporotic 
women demonstrated that the overall prevalence of vitamin D deficiency: serum 25-hydroxy-vitamin D (25[OH]D) $<30 \mathrm{ng} / \mathrm{mL}$, was $64 \%$. In that study, the mean $25(\mathrm{OH}) \mathrm{D}$ concentration was $17.6 \mathrm{ng} / \mathrm{mL}$ in South Korean subjects, and $92 \%$ of them had vitamin D deficiency.[4] Otherwise, there is a lack of studies on vitamin D deficiency in an elderly population in South Korea.

Therefore, we hypothesized that the prevalence of vitamin D deficiency and sarcopenia would be different among particular groups and conducted a prospective study on consecutive elderly (older than 65 years) patients undergoing hip fracture surgery (HFS) versus elective primary total hip arthroplasty (THA) (1) to compare the prevalence of vitamin D deficiency; and (2) to evaluate the association between vitamin $D$ deficiency and sarcopenia.

\section{METHODS}

Among 232 patients who underwent HFS or elective THA by 1 surgeon from March 2017 to April 2018, we included patients who were older than 65 years at the time of surgery and excluded patients who had revision hip arthroplasty, those with metabolic bone disease, chronic renal disease, or rheumatoid arthritis, and those who had a history of calcium, vitamin D, or anti-osteoporosis medication. The most common diagnosis of patients in the elective THA group was the osteonecrosis of the femoral head (54\%) following primary arthritis (21\%) and arthritis due to dysplastic hip (12\%). THA was not performed in HFS group.

This left 170 patients; 70 HFS patients and 100 elective THA patients were subjected to this study. The HFS group included 28 men and 42 women, and the elective THA group included 26 men and 74 women. The mean age at the time of the surgery was 79.8 years vs. 75.4 years, and the mean

Table 1. Patient demographics

\begin{tabular}{lccr}
\hline & $\begin{array}{c}\text { Hip fracture surgery } \\
(\mathrm{N}=70)\end{array}$ & $\begin{array}{l}\text { Total hip arthro- } \\
\text { plasty }(\mathrm{N}=100)\end{array}$ & $P$-value \\
\hline Age $(\mathrm{yr})$ & $79.8 \pm 6.2$ & $74.5 \pm 6.3$ & $<0.001$ \\
Gender & & & 0.066 \\
$\quad$ Male & $28(40.0)$ & $26(26.0)$ & \\
Female & $42(60.0)$ & $74(74.0)$ & \\
Koval score & $2.1 \pm 1.9$ & $2.3 \pm 1.9$ & 0.449 \\
\hline
\end{tabular}

The data is presented as mean \pm standard deviation or number (\%). preoperative Koval score [5] was 2.1 vs. 2.3, respectively (Table 1).

Serum 25(OH)D level was measured by 1125 radioimmunoassay (DIAsource ImmunoAssays SA, Louvain-la-Neuve, Belgium) in the preoperative evaluation.

Vitamin D deficiency was defined as levels less than 10 $\mathrm{ng} / \mathrm{mL}$, and insufficiency as levels between 10 and $30 \mathrm{ng} /$ $\mathrm{mL} .[6]$

Bone mineral density and appendicular skeletal muscle mass (ASM) were measured on dual energy $X$-ray absorptiometry (DXA; QDR 4500A; Hologic Inc., Bedford, MA, USA).

Sarcopenia index was calculated by dividing ASM (kg) by body mass index $\left(\mathrm{kg} / \mathrm{m}^{2}\right)$. A diagnosis of sarcopenia was made when the sarcopenia index was $<0.789$ in males and $<0.512$ in females as described in the Foundation for the National Institutes of Health (FNIH) Sarcopenia Project.[7]

Handgrip power was measured 3 to 5 days after the operation using a dynamometer with patients seated, their elbow flexed to right angles, and a neutral wrist position. For each hand, the mean of three trials of grip strength was calculated.

This study was approved by the Institutional Review Board (IRB) of the Seoul National University Bundang Hospital (IRB no. B-1701/378-303).

\section{Statistical analysis}

Descriptive statistics were performed in SPSS version 16 (IBM Corp., Armonk, NY, USA), and $P$-values of less than 0.05 were considered to indicate statistical significance. The $\chi^{2}$ test was performed for categorical variables, and student's $t$-test was used for continuous variables

Table 2. Comparison of hip fracture surgery patients and total hip arthroplasty patients

\begin{tabular}{lccr}
\hline & $\begin{array}{c}\text { Hip fracture } \\
\text { surgery }\end{array}$ & $\begin{array}{c}\text { Total hip } \\
\text { arthroplasty }\end{array}$ & $P$-value \\
\hline Handgrip power $(\mathrm{kg})$ & $16.9 \pm 7.8$ & $22.8 \pm 13.9$ & 0.007 \\
Vitamin D level $(\mathrm{ng} / \mathrm{mL})$ & $18.6 \pm 12.9$ & $33.3 \pm 17.2$ & $<0.001$ \\
Vitamin D insufficiency $^{\text {a) }}$ & $56(80.0)$ & $34(34.0)$ & $<0.001$ \\
Vitamin D deficiency & $20(29.0)$ & $14(14.0)$ & 0.310 \\
ASM/BMl & $0.65 \pm 0.139$ & $0.62 \pm 0.139$ & 0.097 \\
Sarcopenia & $28(40.0)$ & $36(36.0)$ & 0.632 \\
\hline
\end{tabular}

The data is presented as mean \pm standard deviation or number (\%). a) Vitamin D insufficiency: $<30 \mathrm{ng} / \mathrm{mL}$. b/Vitamin D deficiency: $<10 \mathrm{ng} / \mathrm{mL}$. ASM, appendicular skeletal muscle mass; BMI, body mass index. 
Table 3. Overall comparison of sarcopenia patients and non-sarcopenia patients

\begin{tabular}{lccc}
\hline & $\begin{array}{c}\text { Sarcopenia } \\
(\mathrm{N}=64)\end{array}$ & $\begin{array}{c}\text { Non-sarcopenia } \\
(\mathrm{N}=106)\end{array}$ & P-value \\
\hline Age (yr) & $76.9 \pm 6.6$ & $76.5 \pm 7.0$ & 0.731 \\
Gender & & & 0.001 \\
$\quad$ Female & $34(53.1)$ & $82(77.4)$ & \\
$\quad$ Male & $30(46.9)$ & $24(22.6)$ & \\
Hip fracture surgery & $28(43.8)$ & $42(39.6)$ & 0.623 \\
Total hip arthroplasty & $36(56.3)$ & $64(60.4)$ & \\
Koval score & $2.1 \pm 1.8$ & $2.3 \pm 2.0$ & 0.518 \\
Handgrip power (kg) & $23.5 \pm 16.8$ & $19.4 \pm 9.1$ & 0.061 \\
Vitamin D level (ng/mL) & $28.2 \pm 18.6$ & $27.1 \pm 16.3$ & 0.673 \\
$\quad$ Vitamin D insufficiency & $38(59.4)$ & $52(49.1)$ & 0.208 \\
Vitamin D deficiency ${ }^{\text {b) }}$ & $10(15.6)$ & $24(22.6)$ & 0.325
\end{tabular}

The data is presented as mean \pm standard deviation or number $(\%)$.

a) Vitamin D insufficiency: $<30 \mathrm{ng} / \mathrm{mL}$. ${ }^{b} V$ Vitamin D deficiency: $<10 \mathrm{ng} / \mathrm{mL}$.

\section{RESULTS}

\section{Vitamin D level and prevalence of vitamin D deficiency}

The level of serum vitamin D was lower, and the percentages of vitamin $D$ insufficiency and deficiency patients were higher in the HFS patients compared to those in the THA patients.

There was no significant difference in the prevalence of sarcopenia between 2 groups (Table 2).

When the patients were divided into sarcopenia group (64 patients) and non-sarcopenia group (106 patients), there were no significant differences in Koval score, handgrip power, and serum vitamin D level (Table 3).

\section{Relationship between sarcopenia and vitamin $\mathrm{D}$ deficiency}

Overall, 64 patients $(37.6 \%, 64 / 170)$ were sarcopenic: 28 HFS patients (40\%, 28/70) and 36 THA patients (36\%, 36/100). The level of vitamin $D$ and the percentages of vitamin $D$ insufficiency and deficiency were not significantly different between the sarcopenia group and the non-sarcopenia group (Table 3).

In the HFS patients, sarcopenia was more frequent in men $(57.1 \%, 16 / 28)$ than in women $(28.6 \%, 12 / 42)(P=0.025)$, and vitamin $D$ level was not significantly different between the sarcopenic and non-sarcopenic groups. The prevalence of vitamin D insufficiency was higher in sarcopenic patients
Table 4. Comparison of sarcopenia patients and non-sarcopenia patients among hip fracture patients

\begin{tabular}{lccc}
\hline & $\begin{array}{c}\text { Sarcopenia } \\
(\mathrm{N}=28)\end{array}$ & $\begin{array}{c}\text { Non-sarcopenia } \\
(\mathrm{N}=42)\end{array}$ & $P$-value \\
\hline Age (yr) & $78.3 \pm 5.4$ & $80.8 \pm 6.5$ & 0.092 \\
Gender & & & 0.025 \\
$\quad$ Female & $12(42.9)$ & $30(71.4)$ & \\
$\quad$ Male & $16(57.1)$ & $12(28.6)$ & \\
Koval score & $1.8 \pm 1.7$ & $2.3 \pm 2.0$ & 0.242 \\
Handgrip power (kg) & $17.8 \pm 6.8$ & $16.4 \pm 8.4$ & 0.543 \\
Vitamin D level (ng/mL) & $16.4 \pm 9.4$ & $20.1 \pm 14.7$ & 0.208 \\
$\quad$ Vitamin D insufficiency & $26(92.9)$ & $30(71.4)$ & 0.035 \\
Vitamin D deficiency & $6(21.4)$ & $14(33.3)$ & 0.418 \\
\hline
\end{tabular}

The data is presented as mean \pm standard deviation or number $(\%)$.

${ }^{a}$ Vitamin D insufficiency: $<30 \mathrm{ng} / \mathrm{mL}$. ${ }^{b}$ Vitamin D deficiency: $<10 \mathrm{ng} / \mathrm{mL}$.

$(92.9 \%, 26 / 28)$ than in non-sarcopenic patients $(71.4 \%$, $30 / 42 ; P=0.035$ ) (Table 4).

\section{DISCUSSION}

Hip fractures are the second common osteoporotic fractures next to spinal fractures. Elderly people are prone to have vitamin $D$ deficiency. This is mainly due to reduced sunlight exposure, though decreased capacity for cutaneous production, low dietary intake, poor absorption, and impaired hepatic hydroxylation of vitamin D may also contribute to low 25(OH)D levels.[8] Several epidemiological studies showed that osteomalacia is common in hip fracture patients, but vitamin D levels in these patients were inconsistent.[9]

In our study, $80 \%$ of HFS patients and $36 \%$ of THA patients had vitamin $D$ insufficiency.

The 1,25-dihydroxy-cholecalciferol $\left(1,25[\mathrm{OH}]_{2} \mathrm{D}_{3}\right)$, the active form of vitamin $D$, plays a major role in maintaining neuromuscular function. Previous studies showed that a low level of this hormone results in poor functional level, and hip fracture patients had low serum 1,25(OH)D levels.

Carpintero et al. [10] performed a prospective study in 109 elderly hip fracture patients to verify the relationship between $1,25(\mathrm{OH})_{2} \mathrm{D}_{3}$ levels and functional recovery. In their study, a significant relationship was found between low $1,25(\mathrm{OH})_{2} \mathrm{D}_{3}$ levels and a poor functional recovery 1 year after the fracture.

Vitamin D also has important effects on skeletal muscle. Unlike other authentic vitamins, vitamin $D$ is produced in 
the skin using ultraviolet sunlight. Through its nuclear receptor (i.e., vitamin D receptor) located throughout the muscle, vitamin $D$ regulates myocyte proliferation and growth. In some studies, vitamin D supplementation has been shown to increase muscle strength, particularly in people who have a deficiency of vitamin D.[11]

A recent study from Taiwan to assess the prevalence of vitamin D inadequacy among non-supplemented postmenopausal women with osteoporosis and fragility fractures of the hip or spine, high prevalence of vitamin D deficiency was found across all age groups among non-supplemented women with osteoporosis and fragility fractures.[11]

Conversely, vitamin D appears to play a role in muscle strength, injury prevention, and sports performance, and a significant positive correlation was found between $1,25(\mathrm{OH})_{2} \mathrm{D}_{3}$ levels and muscle strength.[8,12,13]

Even though we could not find an association between vitamin D deficiency and sarcopenia, sarcopenia was frequent in hip fracture patients, especially in men.

There are several limitations of this study. First, the sample size was small. Second, we measured the $25(\mathrm{OH}) \mathrm{D} \mathrm{lev-}$ els at admission. Third, we did not measure parathyroid hormone, which could have contributed to the relationship between 25(OH)D levels and hip fracture. Fourth, the portion of patients who were diagnosed with sarcopenia might have been different if other criteria such as the Asian Working Group for Sarcopenia [14] or the European Working Group on Sarcopenia in Older People [15] were used. Fifth, there might be differences in comorbidities in each group, which should be dealt with in future studies. Sixth, the significant difference in age might obscure the interpretation of results. Further studies using propensity score matching on a large number of patients would be needed.

In conclusion, we found that vitamin D deficiency was more prevalent in hip fracture patients, compared with elective THA patients, and sarcopenia was prevalent especially in men with hip fractures. Recent studies showed that supplementation strategy of vitamin $D$ is effective in decreasing the incidence of osteoporotic fractures in an elderly population.[16,17] We hope that our study would improve our understanding of hypovitaminosis $D$ in hip fracture patients and could guide to prevent osteoporotic fractures.

\section{DECLARATIONS}

\section{Funding}

This study was supported by research funding from Korean Society for Bone and Mineral Research.

\section{Ethics approval and consent to participate}

This study conformed to the ethical guidelines of the 1975 Declaration of Helsinki and was approved by the Institutional Review Board (IRB) of the Seoul National University Bundang Hospital (IRB no. B-1701/378-303).

\section{Conflict of interest}

Young-Kyun Lee has been the associate editor of the Journal of Bone Metabolism since 2009. No potential conflict of interest relevant to this article was reported.

\section{ORCID}

Hong Seok Kim https://orcid.org/0000-0002-9524-7019

Geun Jang

Young-Kyun Lee https://orcid.org/0000-0002-5081-0066

Kyung-Hoi Koo

https://orcid.org/0000-0001-6564-4294

https://orcid.org/0000-0001-5251-2911

\section{REFERENCES}

1. Abrams GD, Feldman D, Safran MR. Effects of vitamin D on skeletal muscle and athletic performance. J Am Acad Orthop Surg 2018;26:278-85. http://dx.doi.org/10.5435/ jaaos-d-16-00464.

2. Bouillon R, Norman AW, Lips P. Vitamin D deficiency. N Engl J Med 2007;357:1980-1; author reply 1-2. http://dx.doi. org/10.1056/NEJMc072359.

3. Kanis JA, Odén A, McCloskey EV, et al. A systematic review of hip fracture incidence and probability of fracture worldwide. Osteoporos Int 2012;23:2239-56. http://dx.doi.org/ 10.1007/s00198-012-1964-3.

4. Hwang YC, Ahn HY, Jeong IK, et al. Optimal serum concentration of 25-hydroxyvitamin $D$ for bone health in older Korean adults. Calcif Tissue Int 2013;92:68-74. http://dx.doi. org/10.1007/s00223-012-9669-3.

5. Koval KJ, Zuckerman JD. Functional recovery after fracture of the hip. J Bone Joint Surg Am 1994;76:751-8. http:// dx.doi.org/10.2106/00004623-199405000-00018.

6. Shin KY, Park KK, Moon SH, et al. Vitamin D deficiency adversely affects early post-operative functional outcomes 
after total knee arthroplasty. Knee Surg Sports Traumatol Arthrosc 2017;25:3424-30. http://dx.doi.org/10.1007/ s00167-016-4209-8.

7. Studenski SA, Peters KW, Alley DE, et al. The FNIH sarcopenia project: rationale, study description, conference recommendations, and final estimates. J Gerontol A Biol Sci Med Sci 2014;69:547-58. http://dx.doi.org/10.1093/gerona/glu010.

8. Lee GH, Lim JW, Park YG, et al. Vitamin D deficiency is highly concomitant but not strong risk factor for mortality in patients aged 50 year and older with hip fracture. J Bone Metab 2015;22:205-9. http://dx.doi.org/10.11005/jbm. 2015.22.4.205.

9. Erem C, Tanakol R, Alagöl F, et al. Relationship of bone turnover parameters, endogenous hormones and vit D deficiency to hip fracture in elderly postmenopausal women. Int J Clin Pract 2002;56:333-7.

10. Carpintero P, Garcia-Lazaro M, Montero M, et al. Relationship between 1,25-dihydroxycholecalciferol levels and functional outcome after hip fracture in elderly patients. Joint Bone Spine 2006;73:729-32. http://dx.doi.org/10.1016/ j.jbspin.2006.01.029.

11. Hwang JS, Tsai KS, Cheng YM, et al. Vitamin D status in non-supplemented postmenopausal Taiwanese women with osteoporosis and fragility fracture. BMC Musculoske- let Disord 2014;15:257. http://dx.doi.org/10.1186/14712474-15-257.

12. Lee JE, Kim KW, Paik NJ, et al. Evaluation of factors influencing grip strength in elderly Koreans. J Bone Metab 2012; 19:103-10. http://dx.doi.org/10.11005/jbm.2012.19.2.103.

13. Johnson AL, Smith JJ, Smith JM, et al. Vitamin D insufficiency in patients with acute hip fractures of all ages and both sexes in a sunny climate. J Orthop Trauma 2013;27:e27580. http://dx.doi.org/10.1097/BOT.0b013e318291f263.

14. Chen LK, Woo J, Assantachai P, et al. Asian Working Group for sarcopenia: 2019 Consensus update on sarcopenia diagnosis and treatment. J Am Med Dir Assoc 2020;21:3007.e2. http://dx.doi.org/10.1016/j.jamda.2019.12.012.

15. Cruz-Jentoft AJ, Bahat G, Bauer J, et al. Sarcopenia: revised European consensus on definition and diagnosis. Age Ageing 2019;48:601. http://dx.doi.org/10.1093/ageing/afz046.

16. Bee CR, Sheerin DV, Wuest TK, et al. Serum vitamin D levels in orthopaedic trauma patients living in the northwestern United States. J Orthop Trauma 2013;27:e103-6. http:// dx.doi.org/10.1097/BOT.0b013e31825cf8fb.

17. Chapuy MC, Pamphile R, Paris E, et al. Combined calcium and vitamin D3 supplementation in elderly women: confirmation of reversal of secondary hyperparathyroidism and hip fracture risk: the Decalyos II study. Osteoporos Int 2002;13:257-64. http://dx.doi.org/10.1007/s001980200023. 
